\title{
When to Suspect Primary Immune Deficiency in Office Practice?
}

\section{Sagar Bhattad*}

Pediatric Immunology and Rheumatology Division, Department of Pediatrics, Aster CMI Hospital, Bangalore, India

*Corresponding Author: Sagar Bhattad, Pediatric Immunology and Rheumatology Division, Department of Pediatrics, Aster CMI Hospital, Bangalore, India.

Received: September 17, 2019; Published: September 30, 2019

DOI: 10.31080/ASPE.2019.02.0157

Keywords: Immune Deficiency; Children; Pediatrics

\section{Introduction}

Primary Immune Deficiencies (PID) are a group of inherited disorders characterized by defect in the immune system that predispose affected individuals to recurrent and/or unusual infections, malignancy, auto-inflammation and autoimmunity. As per an estimate, 1 in 1200 individuals are affected with a PID [1]. However, these diseases are grossly under-diagnosed due to lack of awareness about these diseases amongst pediatricians and physicians.

The International Union of Immunological Societies (IUIS) 2017 classification for PID enlists 330 PIDs [2]. With better understanding of the genetics of immunology and the extensive usage of next generation sequencing, several newer forms of PIDs are being added to this list every year. These disorders are now known as "Inborn Errors of Immunity".

This article would provide a simplified and pragmatic approach to suspected PID. "Eyes see only what mind knows" - Only a physician who is seeking to diagnose PID in his practice, would eventually be able to diagnose these cases. So the first step in the diagnosis of PID is "SUSPICION OF PID".

\section{Warning signs of PID}

Careful clinical evaluation is crucial for identification of PID. Ten warning signs have been suggested by the European Society for Immunodeficiencies(ESID):

1. Four or more new ear infections within 1 year.

2. Two or more serious sinus infections within 1 year.

3. Two or more months on antibiotics with little effect.

4. Two or more pneumonias within 1 year.

5. Failure of an infant to gain weight or grow normally.

6. Recurrent, deep skin or organ abscesses.

7. Persistent thrush in mouth or fungal infection on skin.
8. Need for intravenous antibiotics to clear infections.

9. Two or more deep-seated infections including septicemia. 10. A family history of PID.

Patients showing these signs and symptoms must be subjected to further evaluation to confirm/exclude a PID.

\section{Clinical approach}

While evaluating a child with suspected PID, following factors would assist the treating physician in classifying a child into a broad category of PID.

Age at onset of infections

1. T cell defects (eg: Severe Combined Immune Deficiency) often present in the first 6 months of life.

2. B cell defects (eg: X-linked Agammaglobulinemia) present after 6 months of age, once maternally transmitted IgG levels wean off.

Family history

PIDs are genetic disorders and a detailed family history is of paramount importance in making a timely diagnosis.

1. Consanguinity in parents $\rightarrow$ Autosomal recessive (AR) disease

2. Affected male siblings or death of male siblings/maternal uncle(s) $\rightarrow$ X-linked recessive (XL) disease

3. If one of the parent is affected $\rightarrow$ Autosomal dominant (AD) inheritance

Type of organisms isolated may provide a clue to the underlying PID

1. Fungal infections - Aspergillus; Unusual pathogens - Burkholderia, Serratia, Pseudomonas $\rightarrow$ Chronic granulomatous disease 
2. Encapsulated bacteria - Pneumococcus, H influenza $\rightarrow$ Antibody deficiency (B cell defect), complement deficiency, asplenia

3. Viral, fungal and bacterial infections $\rightarrow \mathrm{T}$ cell defect

4. Atypical mycobacteria - $\mathrm{M}$ abscessus, $\mathrm{M}$ avium etc $\rightarrow$ Mendelian susceptibility to mycobacterial disease

Certain clinical findings can guide a clinician towards a particular PID

1. Chronic eczema + Low platelet counts (in a boy) $\rightarrow$ Wiskott Aldrich syndrome.

2. Cold staphylococcal abscess + Eczema + High IgE $\rightarrow$ AD Hyper IgE syndrome (Jobs syndrome).

3. Extensive molluscum contagiosum + warts + High $\operatorname{IgE} \rightarrow$ DOCK8 deficiency.

4. Delayed cord fall in a neonate + very high neutrophil counts $\rightarrow$ Leucocyte adhesion deficiency (LAD).

\section{Role of routine investigations}

Complete blood counts can provide a clue to the underlying PID

- Persistent lymphopenia in an infant is a marker of Severe Combined Immune Deficiency. Lymphopenia: Absolute lymphocyte count $<3000 / \mathrm{mm} 3$ in an infant.

- Neutropenia (ANC < 1500/mm3) can point towards a PID. Kindly go through Algorithm (F).

- Marked neutrophilia can be seen in LAD.

- $\quad$ Boys presenting with persistent thrombocytopenia must be evaluated for Wiskott Aldrich Syndrome (WAS). One must always look for the mean platelet volume (MPV) in children with thrombocytopenia. Low MPV (small platelets) indicate WAS.

Immunological tests

Commonly used screening tests

1. Immunoglobulin profile (IgG, IgA and IgM) and IgE levels

2. Lymphocyte subsets (T cell, B cell and NK cell counts) and CD4/CD8 counts

3. NBT (nitroblue tetrazoleum) and DHR (dihydrorhodamine dye reduction) tests - to study the phagocytic function.

Algorithms to guide clinicians in the diagnosis of PID

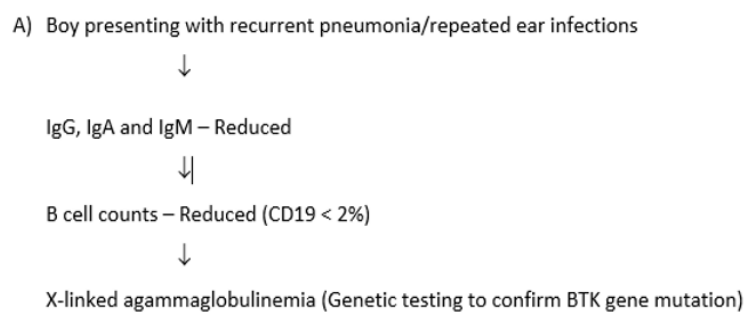

B) Adolescents/ adults presenting with recurrent pneumonia/ repeated ear infections/ recurrent diarrhea

\section{$\downarrow$}

$\operatorname{IgG}, \lg A$ and $\lg M-$ Reduced

$\downarrow$

B cell counts - Normal

$\downarrow$

Common Variable Immune Deficiency (CVID)

C) Suppurative infections (recurrent lymphadenitis/lung abscess/liver abscess) $\downarrow$

$\lg G$, IgA and $\lg M-H i g h$

$\downarrow$

NBT and DHR tests - Abnormal

$\downarrow$

Chronic granulomatous disease (confirm with genetic tests)

D) Boy presenting with recurrent pneumonia/repeated ear infections/eczema/blood in stools



Wiskott Aldrich syndrome (WASP assay by flow cytometry and genetic testing to confirm)

E) Recurrent infections, delayed cord fall, non-healing ulcers

$\downarrow$

Persistently elevated neutrophil counts (ANC $>20,000 / \mathrm{mm}^{3}$, and can be $>100,000 / \mathrm{mm}^{3}$ ) $\downarrow$

Leukocyte adhesion deficiency $\downarrow$

CD18 assay by flow cytometry and confirmation by genetic testing.

F) Recurrent infections, mucositis, oral ulcers

$\downarrow$

Neutropenia $\left(\right.$ ANC $<1500 / \mathrm{mm}^{3}$ )

$\downarrow$

Infants with ANC $<500 / \mathrm{mm}^{3} \quad$ Recurrent neutropenia Immunoglobulins (low IgG) $\downarrow$

$\downarrow$

$\downarrow$

Algorithms 


\section{Conclusion}

Children/adults presenting with recurrent/unusual/persistent/severe infections must be evaluated for underlying PID. HIV infection must be excluded before performing immunological tests. A detailed family history, the type of infections in the affected individual and simple laboratory tests (differential counts, platelet counts) can provide valuable clues to the underlying PID. Serum immunoglobulins must be tested in children with recurrent pneumonia/ear infections/diarrhea. Children with suppurative infections must be screened for phagocytic disorders (NBT, DHR studies). Genetic studies should be performed to confirm the diagnosis and offer genetic counselling. While majority of B cell defects are treated with monthly intravenous immunoglobulin infusions, children with severe $\mathrm{T}$ cell and phagocytic defects need a bone marrow transplant.

\section{Bibliography}

1. Boyle JM and Buckley RH. "Population prevalence of diagnosed primary immunodeficiency diseases in the United States". Journal of Clinical Immunology 27 (2007): 497-502.

2. Bousfiha A., et al. "The 2017 IUIS Phenotypic Classification for Primary Immunodeficiencies". Journal of Clinical Immunology 38 (2018): 129-143.

Volume 2 Issue 10 October 2019

(C) All rights are reserved by Sagar Bhattad. 